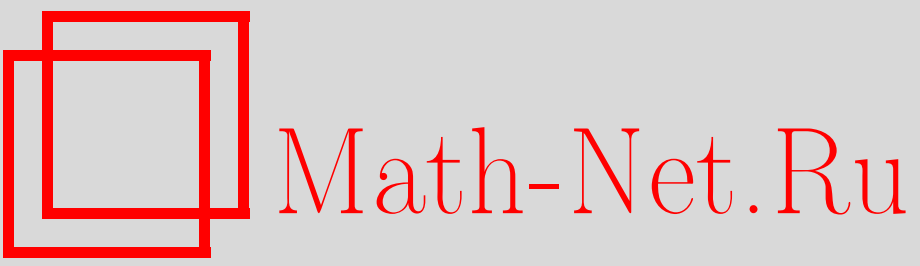

В. И. Денисов, Исследование свойств решения релятивистской теории гравитации в окрестности сингулярной сферы, ТМФ, 1997, том 111, номер 1, 144-148

DOI: https://doi.org/10.4213/tmf996

Использование Общероссийского математического портала Math-Net.Ru подразумевает, что вы прочитали и согласны с пользовательским соглашением

http://www.mathnet.ru/rus/agreement

Параметры загрузки:

IP : 54.237 .59 .107

26 апреля 2023 г., 15:28:46 


\section{ИССЛЕДОВАНИЕ СВОЙСТВ РЕШЕНИЯ РЕЛЯТИВИСТСКОЙ ТЕОРИИ ГРАВИТАЦИИ В ОКРЕСТНОСТИ СИНГУЛЯРНОЙ СФЕРЫ}

Проведено исследование свойств статической сферически-симметричной метрики релятивистской теории гравитации в окрестности сингулярной сферы. Показано, что массивная частица при негеодезическом радиальном движении может достигнуть этой сферы и остаться на ней в покое. На основе этого свойства сделан вывод, что источником сингулярной метрики в релятивистской теории гравитации может служить сфера, созданная из массивных частиц.

Как известно, из-за существенной нелинейности уравнений гравитационного поля в релятивистской теории гравитации точное решение для сферически-симметричного источника до сих пор не найдено. Поэтому в работах $[1,2]$ была получена асимптотика этого решения в предельных случаях сильного и слабого гравитационных полей.

В частности, в работе [2] такое исследование привело к утверж дению о сушествовании в области, близкой к сфере Шварцшильда, сингулярности у метрического тензора эффективного псевдориманова пространства-времени:

$$
\begin{gathered}
g_{00}=U(r)=(1+2 m M) \frac{r-Z_{q}}{r}+q m^{2} M^{2}, \quad q>0, \\
g_{r r}=-V(r)=-\frac{r}{r-Z_{q}} \\
g_{\theta \theta}=-r^{2}, \quad g_{\phi \phi}=-r^{2} \sin ^{2} \theta
\end{gathered}
$$

где $m$ - масса гравитона, $M$ - масса источника гравитационного поля, $Z_{q}=2 M-$ $\nu m^{2} M^{2} \ln m M$, причем $\nu>0$.

Как указывается в работе [3], в области, близкой к сингулярности $r=Z_{q}$, влияние массы гравитона велико и оно принципиально изменяет характер решения по сравнению с решением Шварцшильда в обшей теории относительности. Одним из характерных эффектов, присуших решению (1), является “отскок" свободно падаюших частиш от сферы $r=Z_{q}[1]$.

Опираясь на выражения (1) для статической сферически-симметричной метрики, проведем дальнейший анализ свойств решения релятивистской теории гравитации в

* Московский государственный университет, e-mail: denisov@srdlan.npi.msu.su 
окрестности сингулярной сферы. Выясним условия, при которых массивная частица может приблизиться к сингулярности $r=Z_{q}$ и остаться там в покое.

Несложно показать, что при радиальном движении по геодезической этого достичь не удастся. Действительно, запишем уравнения для радиальной геодезической в метрике (1), обозначая штрихом производную по $r$ и точкой производную по времени $t$ :

$$
\begin{gathered}
\frac{d u^{0}}{d s}+\frac{U^{\prime}}{U} u^{0} u^{r}=0 \\
\frac{d u^{r}}{d s}+\left(u^{0}\right)^{2}\left\{\frac{U^{\prime}}{2 V}+\frac{V^{\prime}}{2 V} \dot{r}^{2}\right\}=0 .
\end{gathered}
$$

Из первого уравнения этой системы следует, что

$$
u^{0}=\frac{E_{0}}{U}
$$

где $E_{0}$ - постоянная интегрирования, зависяшая от начальных значений $r(0)$ и $\dot{r}(0)$ и имеющая смысл начальной координатной энергии частицы, приходящейся на единицу ее массы.

Так как квадрат четырехскорости массивной частищы должен быть равен единище, TO

$$
\dot{r}^{2}=\left\{\frac{1}{U}-\frac{1}{E_{0}^{2}}\right\} \frac{U^{2}}{V} .
$$

Подставляя это равенство во второе уравнение системы (2) и учитывая явный вид (1) функций $U(r)$ и $V(r)$, получим

$$
\ddot{r}=-\frac{(1+2 m M)\left(r-Z_{q}\right) Z_{q}}{2 r^{3}}+\left[1-\frac{U}{E_{0}^{2}}\right]\left\{\frac{(1+2 m M)\left(r-Z_{q}\right) Z_{q}}{r^{3}}+\frac{Z_{q} U}{2 r^{2}}\right\} .
$$

Отсюда непосредственно следует, что радиальное ускорение частищы при $r=Z_{q}$ обрашается в нуль только при выполнении условия

$$
E_{0}^{2}=U\left(Z_{q}\right)=q m^{2} M^{2}
$$

Несложно убедиться, что для свободно падаюшей по радиусу частицы это условие не может быть обеспечено. Действительно, если бы это условие выполнялось, то квадрат радиальной скорости частицы (3) был бы отрицателен при $r>Z_{q}$ :

$$
\dot{r}^{2}=\left\{\frac{1}{U}-\frac{1}{U\left(Z_{q}\right)}\right\} \frac{U^{2}}{V}<0
$$

в силу того что $U(r)>U\left(Z_{q}\right)$.

Поэтому на свободно падаюшую вдоль радиуса частицу в окрестности сферы Шварцшильда действует сила отталкивания, которая сначала останавливает ее, после чего частица начинает двигаться от центра, т.е. происходит эффект “отскока”. 
Покажем теперь, что при негеодезическом радиальном движении массивной частицы можно добиться того, что частица, достигнув сферы $r=Z_{q}$, останется там в покое, и выясним, какие требования накладывает это условие на силу негравитационной природы.

Для этого предположим, что на радиально движушуюся частицу, имеющую массу $m_{0}$, действует тормозящая сила негравитационной природы, направленная по радиусу (сила трения или, например, сила, создаваемая реактивным двигателем). В этом случае уравнения движения частицы примут вид

$$
\begin{gathered}
\frac{d u^{0}}{d s}+\frac{U^{\prime}}{U} u^{0} u^{r}=\frac{F^{0}}{m_{0}}, \\
\frac{d u^{r}}{d s}+\left(u^{0}\right)^{2}\left\{\frac{U^{\prime}}{2 V}+\frac{V^{\prime}}{2 V} \dot{r}^{2}\right\}=\frac{F^{r}}{m_{0}},
\end{gathered}
$$

где $F^{0}$ и $F^{r}$ - компоненты четырехвектора силы негравитационной природы.

Построим выражение для этой силы. Так как четырехвектор силы должен быть ортогонален четырехвектору скорости частицы $F^{i} u_{i}=0$, то

$$
F^{0}=\frac{F^{r} u^{r} V}{u^{0} U}=\frac{\dot{r} F^{r} V}{U} .
$$

Подставляя это равенство в первое уравнение системы $(3)$ и умножая его на $U$, получим

$$
\frac{d}{d s}\left(U u^{0}\right)=\frac{F^{r} u^{r} V}{m_{0} u^{0}}
$$

Если теперь потребовать, чтобы радиальная компонента четырехвектора силы удовлетворяла условию

$$
F^{r}=\frac{m_{0} u^{0} Q^{\prime}}{V},
$$

где $Q=Q(r)$ - пока произвольная функция, то уравнение (5) примет вид

$$
\frac{d}{d s}\left(U u^{0}\right)=\frac{d}{d s}(Q) .
$$

Отсюда следует, что

$$
U u^{0}-Q=A_{0}=\text { const }
$$

Таким образом,

$$
u^{0}=\frac{A_{0}+Q}{U}
$$

Так как $u^{i} u_{i}=1$, то

$$
\dot{r}^{2}=\left\{\frac{1}{U}-\frac{1}{\left(Q+A_{0}\right)^{2}}\right\} \frac{U^{2}}{V} .
$$

Сравнивая это равенство с аналогичным равенством (3) геодезического движения, видим, что постоянная $E_{0}$ перешла в некоторую функцию координат $Q(r)+A_{0}$. 
Радиальное ускорение частицы можно найти из второго уравнения системы (4). Учитьвая выражения (6)-(8), в результате будем иметь

$$
\ddot{r}=\left[\frac{U^{\prime}}{U}-\frac{V^{\prime}}{2 V}-\frac{Q^{\prime}}{\left(A_{0}+Q\right)}\right]\left[\frac{1}{U}-\frac{1}{\left(Q+A_{0}\right)^{2}}\right] \frac{U^{2}}{V}-\frac{U^{\prime}}{2 V}+\frac{U Q^{\prime}}{V\left(Q+A_{0}\right)} .
$$

Для того чтобы материальная точка достигла сферы $r=Z_{q}$ и осталась покоиться на этой сфере, необходимо потребовать, чтобы при $r=Z_{q}$ выполнялись два условия: $\dot{r}\left(Z_{q}\right)=0, \ddot{r}\left(Z_{q}\right)=0$. Используя явные выражения (1) для функций $U$ и $V$, несложно установить, что эти условия выполняются, если $Q\left(Z_{q}\right)=\sqrt{U\left(Z_{q}\right)}-A_{0}$.

Выясним теперь, при каком условии время движения частицы к сфере $r=Z_{q}$ будет конечным, если она начала двигаться с некоторого конечного значения $r(0)>Z_{q}$. Положим, что

$$
Q(r)=\sqrt{U(r)}-A_{0}+\left(r-Z_{q}\right)^{\alpha} f(r),
$$

где $f(r)$ - ограниченная знакоположительная функция при $r \geq Z_{q}$ и $\alpha$ - некоторьй положительный параметр. Тогда из выражения (8) получим

$$
\left(\frac{d r}{d t}\right)^{2}=\frac{\left(r-Z_{q}\right)^{2 \alpha+1} U f^{2}(r)+2\left(r-Z_{q}\right)^{\alpha+1} \sqrt{U^{3}} f(r)}{r\left[\sqrt{U}+\left(r-Z_{q}\right)^{\alpha} f(r)\right]^{2}} .
$$

Из общей теории [4] следует, что время достижения точки $r=Z_{q}$ будет конечным, если $\alpha<1 / 2$. Если же $\alpha>1 / 2$, то время достижения сферы $r=Z_{q}$ будет бесконечным и частица лишь асимптотически, при $t \rightarrow \infty$ достигнет сферы $r=Z_{q}$. Так как компонента $g_{00}$ метрического тензора (1) при $r=Z_{q}$ не обращается в нуль, то эти утверждения сохраняют свою силу и при измерении времени по собственным часам частицы.

Из выражений (6)-(8) и (10) следует, что радиальная компонента силы негравитационной природы $F^{r}$ при $0<\alpha<1 / 2$ обрашается в нуль на сфере $r=Z_{q}$. Так как и ускорение частицы (9), и ее скорость (11) при $r=Z_{q}$ равны нулю, то это означает, что материальная частица после достижения сферы $r=Z_{q}$ будет в дальнейшем покоиться на этой сффере.

Таким образом, если создать бесконечно тонкую сферу из массивных частиц суммарной массы $M$ и заставить их двигаться к центру при наличии силы негравитационной природы, то можно добиться, что за конечный промежуток времени размер этой материальной сферы уменьшится до радиуса $R=Z_{q}$. Так как после этого все частищы сферы будут иметь нулевые радиальные скорость и ускорение, то эта сфера в дальнейшем будет находиться в статическом состоянии.

В результате эта бесконечно тонкая материальная сфера будет являться источником статического сферически-симметричного поля, которое в ее окрестности имеет вид (1). $\mathrm{K}$ сожалению, из-за бесконечной плотности вещества $\left(\rho(r) \sim \delta\left(r-Z_{q}\right)\right)$ на сфере $r=Z_{q}$ и невозможности сшивания внешнего $r>Z_{q}$ и внутреннего $r<Z_{q}$ решений (у некоторых компонент метрического тензора $g_{i k}$ обязан быть скачок при $r=Z_{q}$ из-за бесконечной плотности вещества на этой сфере) сделать какие-либо утверждения о метрическом тензоре внутри массивной сферы не представляется возможным.

В заключение автор выражает глубокую благодарность академику А. А. Логунову за постановку задачи и полезные обсуждения. 


\section{Список литературы}

[1] А.А. Власов, А.А. Логунов. ТМФ. 1989. Т. 78. С. 323.

[2] Ю. М. Лоскутов. ТМФ. 1990. Т. 82. С. 304.

[3] А. А. Логунов. Релятивистская теория гравитации и принцип Маха. Препринт ИФВЭ 95-128, 1995.

[4] В. В. Петкевич. Теоретическая механика. М.: Наука, 1981. С. 80.

Поступила в редакцию 14.X.1996 г.

\section{V.I. Denissov \\ ANALYSIS OF THE SOLUTION PROPERTIES OF THE RELATIVISTIC THEORY OF GRAVITATION IN THE VICINITY OF A SINGULAR SPHERE}

The analysis of the properties of static spherical symmetric metric of the relativistic theory of gravitation in the vicinity of a singular sphere is done. It is shown that a massive particle in the nongeodesical radial motion may reach this sphere and be remained there in the rest. Basing on this property, it is inferred that the sphere formed by the massive particles can serve as the source of a singular metic in the relativistic theory of gravitation. 